\title{
Physical literacy levels of Canadian children aged 8-12 years: descriptive and normative results from the RBC Learn to Play-CAPL project
}

Mark S. Tremblay ${ }^{1 *}$, Patricia E. Longmuir ${ }^{1}$, Joel D. Barnes ${ }^{1}$, Kevin Belanger $^{1}$, Kristal D. Anderson ${ }^{2}$, Brenda Bruner ${ }^{3}$, Jennifer L. Copeland ${ }^{4}$, Christine Delisle Nyström¹, Melanie J. Gregg ${ }^{5}$, Nathan Hall', Angela M. Kolen ${ }^{6}$, Kirstin N. Lane ${ }^{2}$, Barbi Law ${ }^{3}$, Dany J. MacDonald7, Luc J. Martin ${ }^{8}$, Travis J. Saunders ${ }^{7}$, Dwayne Sheehan 9 , Michelle R. Stone ${ }^{10}$ and Sarah J. Woodruff ${ }^{11}$

\begin{abstract}
Background: The current physical literacy level of Canadian children is unknown. The Royal Bank of Canada (RBC) Learn to Play - Canadian Assessment of Physical Literacy (CAPL) project, which is anchored in the Canadian consensus statement definition of physical literacy, aimed to help establish the current physical literacy level of Canadian children.

Methods: The CAPL was used to assess the physical literacy (and component domains: Daily Behaviour, Physical Competence, Knowledge and Understanding, and Motivation and Confidence) of Canadian children aged 8-12 years. Data were collected from 11 sites across Canada, yielding a sample of 10,034 participants (5030 girls). Descriptive statistics by age and gender were calculated and percentile distributions of physical literacy scores, including each domain and individual measure, were derived.

Results: The mean age of participants was $10.1 \pm 1.2$ years. Total physical literacy scores (out of 100) were on average $63.1 \pm 13.0$ for boys and $62.2 \pm 11.3$ for girls. For boys and girls respectively, domain scores were $19.9 \pm 4.7$ and $19.3 \pm 4.1$ (out of 32) for Physical Competence; $18.6 \pm 7.9$ and $18.5 \pm 7.4$ (out of 32) for Daily Behaviour; $12.7 \pm 2.8$ and $12.2 \pm 2.6$ (out of 18) for Motivation and Confidence; and 11.8 \pm 2.8 and 12.2 \pm 2.6 (out of 18) for Knowledge and Understanding. Physical Competence measures were on average $28.1 \pm 8.4 \mathrm{~cm}$ (sit-and-reach flexibility), $33.5 \pm$ $9.4 \mathrm{~kg}$ (grip strength, right + left), $23.4 \pm 14.1$ laps (Progressive Aerobic Cardiovascular Endurance Run [PACER] shuttle run), $61.8 \pm 43.8 \mathrm{~s}$ (isometric plank), $19.0 \pm 3.8 \mathrm{~kg} / \mathrm{m}^{2}$ (body mass index), $67.3 \pm 10.8 \mathrm{~cm}$ (waist circumference), and $20.6 \pm 3.9$ out of 28 points for the Canadian Agility and Movement Skill Assessment (CAMSA), with scores for boys higher than girls and older children higher than younger children for grip strength, PACER, plank, and CAMSA score. Girls and younger children had better scores on the sit-and-reach flexibility than boys and older children. Daily pedometer step counts were higher in boys than girls (12,355 \pm 4252 vs. 10,779 \pm 3624$)$, and decreased with age.

\footnotetext{
* Correspondence: mtremblay@cheo.on.ca

${ }^{1}$ Healthy Active Living and Obesity Research Group, Children's Hospital of Eastern Ontario Research Institute, 401 Smyth Road, Ottawa, ON K1H 8L1, Canada

Full list of author information is available at the end of the article
}

(c) The Author(s). 2018 Open Access This article is distributed under the terms of the Creative Commons Attribution 4.0 International License (http://creativecommons.org/licenses/by/4.0/), which permits unrestricted use, distribution, and reproduction in any medium, provided you give appropriate credit to the original author(s) and the source, provide a link to the Creative Commons license, and indicate if changes were made. The Creative Commons Public Domain Dedication waiver (http://creativecommons.org/publicdomain/zero/1.0/) applies to the data made available in this article, unless otherwise stated. 
(Continued from previous page)

Conclusions: These results provide the largest and most comprehensive assessment of physical literacy of Canadian children to date, providing a "state of the nation" baseline, and can be used to monitor changes and inform intervention strategies going forward.

Keywords: Daily behaviour, Physical competence, Knowledge, Understanding, Motivation, Confidence, Physical fitness, Motor skill, Physical activity

\section{Background}

Physical literacy is defined in this paper as the "motivation, confidence, physical competence, knowledge and understanding to value and take responsibility for engagement in physical activities for life" [1]. Interest in physical literacy has increased rapidly in recent years and programs, curricula, and policies intended to improve physical literacy are emerging, with some researchers and educators articulating that physical literacy is as important to develop as literacy and numeracy [2-4]. This is logical given the favourable associations between physically active lifestyles and a wide variety of health indicators [5]. Several countries have begun to incorporate the construct of physical literacy into their educational systems $[1,4,6]$; however, a global consensus on the definition of physical literacy is still lacking [7], and a recent systematic review concluded that little empirical research assessing physical literacy has been conducted to date [8]. Due to the limited amount of objective physical literacy data, the Canadian Assessment of Physical Literacy (CAPL) was developed.

The CAPL was developed and refined between 2009 and 2013, and its overall aim is to provide a reliable, feasible, and valid instrument to assess physical literacy in Canadian children $[2,9,10]$. It incorporates 25 measures within four interrelated domains: Physical Competence, Daily Behaviour, Knowledge and Understanding, and Motivation and Confidence. The CAPL scoring system was developed using a Delphi process with international experts in various fields representing the four domains [11]. An overall physical literacy score (out of 100) as well as individual domain scores are calculated using the CAPL. As advised by the Delphi expert panel, the Physical Competence and Daily Behaviour domains (each maximum 32 points) are weighted higher than the Knowledge and Understanding and the Motivation and Confidence domains (each maximum 18 points) due to the fact that the former are easier to assess objectively $[9,11]$.

Assessments and evaluation are very important in the education and health fields [12], with the assessment of physical fitness in North American children gaining prominence approximately 60 years ago [13]. For the past several decades there has been a contentious debate regarding the methods and appropriateness of physical fitness testing $[14,15]$. The declining physical fitness levels in Canadian children [16] is concerning, as physical fitness has been found to be a powerful marker of health in children and adolescents [17]. Due to the controversy surrounding the assessment of physical fitness in children and youth, Lloyd et al. [12] suggested that instead of measuring only one component (i.e., physical fitness) in physical education classes, we should instead be assessing physical literacy, a broader, more holistic construct. As little is known regarding the physical literacy levels of Canadian children to date, and as monitoring and surveillance are required to assess interventions and trends, the aim of this paper was to establish the current physical literacy levels of Canadian children aged 8 to 12 years using the CAPL.

\section{Methods}

\section{Study design}

The Royal Bank of Canada Learn to Play - Canadian Assessment of Physical Literacy (RBC Learn to PlayCAPL) was a national, multi-site surveillance research project that investigated the physical literacy levels of Canadian children aged 8-12 years. This study collected cross-sectional data from 2014 to 2017 through convenience sampling methods, using study sites identified and selected through professional networks of the RBC Learn to Play-CAPL Principal Investigator (PI) while being attentive to geographic dispersion. The coordinating centre for the RBC Learn to Play-CAPL study was the Healthy Active Living and Obesity Research Group located at the Children's Hospital of Eastern Ontario Research Institute (CHEO RI). The overall study protocol was initially approved by the CHEO Research Ethics Board. Each participating study site was subsequently required to obtain research ethics approval from their respective institution, as well as approval from local school boards and individual schools or organizations where data collection occurred. All children participating in the study provided verbal assent, and parents or legal guardians provided their written informed consent, before data collection began.

\section{Study sites}

Eleven study sites (including the coordinating centre), geographically dispersed across seven Canadian provinces, were selected to participate in the RBC Learn to 
Play-CAPL study: Victoria, British Columbia; Calgary, Alberta; Lethbridge, Alberta; Winnipeg, Manitoba; Windsor, Ontario; North Bay, Ontario; Ottawa, Ontario (coordinating centre); Trois-Rivières, Québec; Halifax, Nova Scotia; Antigonish, Nova Scotia; and Charlottetown, Prince Edward Island. All study site PIs were brought in to the coordinating centre for a two-day standardized training workshop on the CAPL's background, how the measures were to be administered, and how data were to be collected and entered; study site PIs were then able to practise the CAPL on a subset of participants not included in these analyses. Study site PIs then trained Research Assistants and undergraduate/ graduate level students from their institutions as a data collection team. The goal was to collect data on 10,000 children distributed across participating sites.

\section{Participants and setting}

Canadian children aged 8-12 years were recruited for the RBC Learn to Play-CAPL study. This specific age range was selected since the CAPL was initially designed for this age range and has undergone either validity and/ or reliability testing only for children 8-12 years old [10]. Convenience sampling techniques (e.g., established contacts or relationships; schools that volunteered after being contacted by school boards; schools/summer camps in reasonable proximity) were used; children were predominantly recruited through elementary schools in urban, rural, and suburban locations, to ensure that children of different socioeconomic status and physical activity levels were reached. In order to meet the target sample size, sites were encouraged to approach munici$\mathrm{pal} /$ community organizations, after-school care services, family programs, and summer camps. Elite sport teams were not targeted for data collection, as these groups were not representative of the population at large.

\section{CAPL scoring and measures}

The CAPL is comprised of domains and measures that align with the Canadian consensus and International Physical Literacy Association definitions of physical literacy [18]. The CAPL's four domains are Daily Behaviour, Physical Competence, Knowledge and Understanding, and Motivation and Confidence. Daily Behaviour (32 points) and Physical Competence (32 points) were assigned more weight in the overall CAPL score (out of 100) than Knowledge and Understanding (18 points) and Motivation and Confidence (18 points), based on existing theoretical frameworks and input from the Delphi process (see Fig. 1) [11]. For each individual measure, domain score, and overall CAPL score, children are assigned one of four interpretation categories (stratified by age and gender) based on their performance: beginning, progressing, achieving, or excelling (see Additional file 1).
The measures within the CAPL are standardized; whereby instructions, delivery, and scoring are uniform across testers and their respective study sites. Detailed descriptions of each measure are available online in the CAPL manual (https://www.capl-ecsfp.ca).

\section{Daily behaviour}

Children's physical activity levels were both objectively measured and self-reported in the CAPL, as per input from experts participating in the tool's development Delphi process [11]. Physical activity was objectively measured by an SC-StepRx pedometer (StepsCount, Deep River, ON, Canada). Children were instructed to wear the pedometer around their waist on the right hip for seven days (beginning the day after the pedometers were distributed by the research staff), and to complete a daily $\log$ sheet indicating the time the pedometer was put on in the morning, the time it was taken off at night, and if the pedometer was removed for any reason (e.g., swimming, bathing). For self-reported weekly physical activity levels, children were asked, "During the past week (seven days), on how many days were you physically active for a total of at least 60 minutes per day?" Response options ranged from 0 days to 7 days.

In addition to physical activity levels, children were asked to report their time spent in various sedentary behaviours. Participants were asked to self-report their time spent watching $\mathrm{TV}$, playing video or computer games, using a computer for non-school work, and time spent sitting down doing non-screen-based activities outside of school time (e.g., reading a book, doing homework). Response options for each question were: "I did not spend time", "Less than 1 hour", " 1 h", " 2 h", " 3 h", " $4 \mathrm{~h}$ ", and "5 or more hours". Each question was asked for a typical school day and a typical weekend day. The sedentary behaviour questions, and the self-reported physical activity question, were based on the Youth Risk Behavior Surveillance System [19].

\section{Physical competence}

The Physical Competence domain assesses the musculoskeletal fitness, motor competence, and anthropometric characteristics of the child. Muscular strength was assessed by a Smedley III Analog Grip Strength Dynamometer (Creative Health Products, Ann Arbor, MI, USA). While standing, children were instructed to grasp the dynamometer with one hand and abduct that arm away from their torso (approximately 30-45 degrees). While keeping the elbow straight, children were told to "squeeze" the dynamometer handle by performing a fist motion. Children performed two trials with each hand, alternating between hands for each trial, and the maximum scores for each hand $(\mathrm{kg})$ were combined to calculate the total score [16]. 


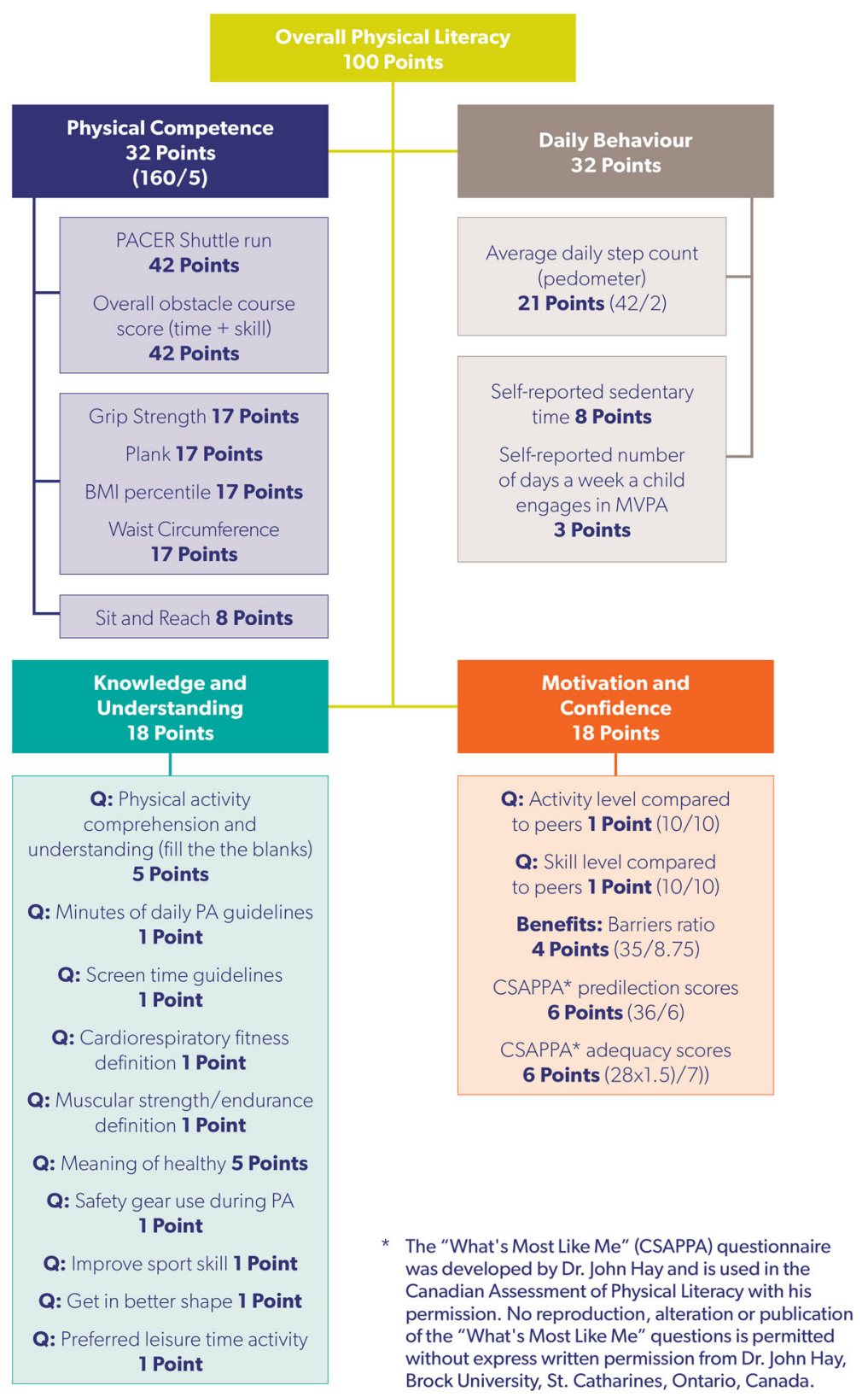

Fig. 1 Canadian Assessment of Physical Literacy scoring system. BMI: body mass index; CSAPPA: Children's Self-Perception of Adequacy in and Predilection for Physical Activity; MVPA: moderate- to vigorous-intensity physical activity; PA: physical activity; PACER: Progressive Aerobic Cardiovascular Endurance Run

Trunk muscular endurance was measured using the isometric torso plank protocol [20]. Children were instructed to assume the plank position (i.e., push-up position but using their forearms for support instead of their palms) and maintain the isometric position for as long as they could without breaking form. Children were allowed one correction by the research staff to resume proper form. The measure concluded when the child either displayed volitional fatigue (e.g., dropping to their knees) or when a second break in form was observed.
Only one trial was conducted and scores were recorded to the nearest $0.1 \mathrm{~s}$.

Aerobic fitness was assessed using the Progressive Aerobic Cardiovascular Endurance Run (PACER) [21]. For the PACER, a progressive test, children and youth were asked to run back and forth between two parallel lines $20 \mathrm{~m}$ apart or $15 \mathrm{~m}$ apart (subsequently converted to 20-m distance score, according to Carrel et al. [22]). An audio recording paced the participants, beginning at a speed of $8.5 \mathrm{~km} / \mathrm{h}$ and increasing by $0.5 \mathrm{~km} / \mathrm{h}$ every 
consecutive minute. Participants continued until they were no longer able to keep pace with the audio recording for two consecutive laps, at which point their last completed lap was recorded.

Motor competence was evaluated using the Canadian Agility and Movement Skill Assessment (CAMSA), which is an obstacle-type course that combines both fundamental (jumping, sliding, catching, throwing, etc.) and complex (acceleration, deceleration, hand-eye coordination, etc.) movement skills [23]. Each child performed four trials on the CAMSA: two practice trials and two test trials. Children were scored on time (nearest $0.1 \mathrm{~s}$ ) required to complete the CAMSA (range 1-14 points) and their ability to demonstrate the movement skill criteria (range 0-14 points) for a combined score out of 28. The best score out of the two test trials was used for CAPL scoring. The CAMSA has been shown to have good convergent validity (older age and boys achieved a higher total score); good inter-rater reliability evidence (intraclass correlation coefficient $[\mathrm{ICC}]=0.99$ for completion time and substantial for skill score ICC $=0.69)$; and moderate intra-rater reliability $(\mathrm{ICC}=0.52)$ for skill score and excellent reliability for completion time $(\mathrm{ICC}=0.99)$. Reliability was also excellent for completion time over a short $(2-4$ days; ICC $=0.84)$ or long $(8-14$ days; $I C C=0.82)$ interval, while skill score reliability was moderate $(\mathrm{ICC}=0.46)$ over a short interval, and substantial $(\mathrm{ICC}=0.74)$ over a long interval [24].

Static flexibility was assessed using the sit-and-reach protocol [16]. Children were instructed to sit on a floor mat with their legs fully extended in front of them, and the balls of their feet touching the Novel Acuflex I flexometer (Creative Health Products, Ann Arbor, MI, USA). While keeping their legs extended, children were advised to extend both arms toward their toes and stack their hands on top of one another. Children were then instructed to reach forward by performing trunk flexion and push the metal tracker on the flexometer as far as possible, holding the final end-point for five seconds. Children performed two trials, and the score from the best trial (nearest $0.5 \mathrm{~cm}$ ) was used for CAPL scoring.

Height was measured in duplicate with a portable stadiometer (SECA, Hamburg, Germany) without footwear to the nearest $0.1 \mathrm{~cm}$, and weight was measured with a digital scale (A\&D Medical, Milpitas, CA, USA) or mechanical beam scale (if used weight was measured in duplicate) without footwear to the nearest $0.5 \mathrm{~kg}$ [16]. Body mass index (BMI) was calculated by dividing the child's weight in kilograms by their height in metres squared, and converted to a BMI z-score using the World Health Organization (WHO)'s BMI-for-age charts and formulae based on the LMS method [25]. Waist circumference was measured in duplicate using a non-elastic tape measure at the level of the iliac crest and recorded to the nearest $0.5 \mathrm{~cm}$, with the average of the two measures used for analyses [16]. If duplicate measures varied by greater than $0.5 \mathrm{~cm}$ or $0.5 \mathrm{~kg}$ for the aforementioned protocols, a third measure was taken and an average of the closest two measures was recorded.

\section{Knowledge and understanding}

Children completed a 10-indicator Physical Literacy Knowledge Questionnaire, either in paper-and-pencil format or online through the CAPL website, to assess their knowledge and understanding of items related to physical activity. The questions were anchored in Canadian provincial curricula for physical and health education for children in grades 4 to 6 [26]. Children answered questions on a variety of topics, including knowledge of the Canadian Physical Activity Guidelines for Children and Youth [27], knowledge of the Canadian Sedentary Behaviour Guidelines for Children and Youth [28], knowledge of the terms "cardiorespiratory fitness" and "muscular strength", knowledge of "what it means to be healthy" (matching the word "healthy" to various phrases), a comprehension and understanding paragraph (fill in the blanks with a word bank provided), knowledge of when to use safety equipment during activities (circling activities that are performed by the child and determining whether or not safety gear is needed for those activities), knowledge on how to improve sport skills and fitness, and responding to a question on their preferred leisure time activities (either active or inactive pursuits). The Physical Literacy Knowledge Questionnaire demonstrates good validity and feasibility in this age group and is available elsewhere in this supplement [26]. Knowledge scores increased with age (partial eta ${ }^{2}=0.07$ ) but were not related to gender, supporting the validity of the questionnaire. Test-retest reliability for the questionnaire score and individual questions was substantial to excellent for $71 \%$ of comparisons over a 2-day interval, but lower over a 7-day interval (53\% substantial or excellent). More details on the Physical Literacy Knowledge Questionnaire are available in an accompanying manuscript [26].

\section{Motivation and confidence}

Children completed a five-indicator questionnaire, either in paper-and-pencil format or online through the CAPL website, to assess their motivation and confidence levels for physical activity. Children answered questions on a variety of motivation- and/or confidence-related constructs: a benefits-to-barriers ratio for physical activity was calculated from children rating their agreement on a scale of 1 to 5 ( $1=$ disagree; $5=$ agree $)$ for 19 proposed items (10 barriers and nine benefits) [29]; adequacy and predilection subscales representing 17 items from the Children's Self-Perceptions of Adequacy in, and Predilection for Physical Activity (CSAPPA) Scale were determined by children answering items using a structured 
alternative response format (scale of 1 to 4) [30]; and "activity levels compared to others" and "skill level compared to others" were determined by children completing one item for each construct using a 10-point scale ( 1 = "a lot less active" OR "others are better"; $10=$ "a lot more active" OR "I'm a lot better"). The Adequacy and Predilection subscales have been shown to have good test-retest reliability and predictive validity [30].

\section{Paradata}

Given the novel nature of this research, efforts were built into the larger study to better understand the consequences (e.g., refusals, adverse events) and inclusiveness of collecting physical literacy surveillance data. Accordingly, in a subset of 510 participants from six sites, we assessed refusal rates across the various measures. Among a subset of 1196 participants with detailed participation data, the prevalence and type of reported disabilities or medical conditions identified by parents was examined. Records of all 10,034 participants were checked for reporting of adverse events.

\section{Statistical analysis}

Means and standard deviations were calculated by age and gender for all CAPL variables. Generalized additive models for location, scale, and shape (GAMLSS) were used to generate normative values for several variables in the RBC Learn to Play-CAPL dataset. GAMLSS models use different methods to treat over-dispersion, skewness, and kurtosis in a dependent variable within a univariate analysis as compared to more traditional regression models. The "gamlss" R package was used to fit the GAMLSS models [31]. Effect size differences between boys and girls were examined using the Cohen's $d$ method [32], reflecting the magnitude of the difference between groups. To examine differences across ages, unstandardized beta estimates from linear regression models regressing the variable of interest on age in years were divided by their standard deviation to provide the average effect size across age. Effect sizes were considered negligible if $<0.2$, small if between 0.2 and 0.5 , moderate if between 0.5 and 0.8 , and important if $>0.8$ [32]. All analyses were performed using R 3.5.0 (The $R$ Foundation for Statistical Computing, Vienna, Austria).

\section{Results}

A total of 10,034 children participated in the RBC Learn to Play-CAPL project. Table 1 shows the breakdown of the participants by gender and data collection site. Table 2 displays the RBC Learn to Play-CAPL descriptive statistics overall and stratified by gender. Overall, the mean age of the participants was $10.1 \pm 1.2$ years, with $50.1 \%(n=5030)$ of the participants being girls.
Table 1 The number of study participants by gender and data collection site for the RBC Learn to Play-CAPL project

\begin{tabular}{lllll}
\hline Site (city, province) & $\begin{array}{l}\text { Number of } \\
\text { schools/camps }\end{array}$ & Boys & Girls & Total \\
\hline Antigonish, NS & 11 & 515 & 549 & 1064 \\
Calgary, AB & 2 & 637 & 633 & 1270 \\
Charlottetown, PEI & 17 & 269 & 267 & 536 \\
Halifax, NS & 20 & 424 & 431 & 855 \\
Lethbridge, AB & 18 & 551 & 564 & 1115 \\
North Bay, ON & 20 & 533 & 589 & 1122 \\
Ottawa, ON & 43 & 430 & 448 & 878 \\
Trois-Rivières, QC & 3 & 67 & 48 & 115 \\
Victoria, BC & 6 & 268 & 231 & 499 \\
Windsor, ON & 29 & 670 & 608 & 1278 \\
Winnipeg, MB & 16 & 640 & 662 & 1302 \\
Total & 185 & 5004 & 5030 & 10034 \\
\hline AB Albea BC Bitish Coln & & &
\end{tabular}

$A B$ Alberta, $B C$ British Columbia, CAPL Canadian Assessment of Physical Literacy, MB Manitoba, NS Nova Scotia, ON Ontario, PEI Prince Edward Island, RBC Royal Bank of Canada, QC Québec

Total physical literacy scores (out of 100) were on average $63.1 \pm 13.0$ for boys and $62.2 \pm 11.3$ for girls. For boys and girls respectively, domain scores were: $19.9 \pm$ 4.7 and $19.3 \pm 4.1$ for Physical Competence (out of 32 points); $18.6 \pm 7.9$ and $18.5 \pm 7.4$ for Daily Behaviour (out of 32 points); $12.7 \pm 2.8$ and $12.2 \pm 2.6$ for Motivation and Confidence (out of 18 points); and $11.8 \pm 2.8$ and $12.2 \pm 2.6$ for Knowledge and Understanding (out of 18 points). For the total physical literacy score and the domain scores there were negligible differences observed between genders (Cohen's $d$ range: 0.02 to 0.18 ). Using the CAPL's interpretation system (which divides the participants into four categories: beginning, progressing, achieving, and excelling), based on the average total physical literacy score, both boys and girls would be classified as progressing. The individual domain scores for both genders would be classified as progressing for the Physical Competence, Daily Behaviour, and Motivation and Confidence domains, whereas they would be classified as achieving in the Knowledge and Understanding domain. The proportion of participants in each of the four interpretation categories by domain are presented in Fig. 2.

Table 3 shows the RBC Learn to Play-CAPL overall descriptive statistics stratified by age (in one-year increments). The total physical literacy score, as well as the domain scores for Physical Competence and for Knowledge and Understanding, increased with age. The Daily Behaviour domain score decreased with age (effect size negligible; Daily Behaviour domain score decreased by 0.65 units on average as age increased by one year), whereas the Motivation and Confidence domain score exhibited no age-related differences. 
Table 2 RBC Learn to Play-CAPL descriptive statistics, overall and by gender

\begin{tabular}{|c|c|c|c|c|c|c|c|c|}
\hline \multirow[t]{2}{*}{ Variable } & \multicolumn{2}{|l|}{ Overall } & \multicolumn{2}{|l|}{ Boys } & \multicolumn{2}{|l|}{ Girls } & \multicolumn{2}{|c|}{ Boys vs. girls } \\
\hline & $n$ & Mean \pm SD & $n$ & Mean \pm SD & $n$ & Mean \pm SD & Cohen's $d$ & $95 \% \mathrm{Cl}$ \\
\hline Age (years) & 10,034 & $10.1 \pm 1.2$ & 5004 & $10.1 \pm 1.2$ & 5030 & $10.1 \pm 1.2$ & -0.01 & $-0.05,0.03$ \\
\hline Physical Competence score (/32) & 9388 & $19.6 \pm 4.4$ & 4687 & $19.9 \pm 4.7$ & 4701 & $19.3 \pm 4.1$ & 0.14 & $0.10,0.18$ \\
\hline Sit-and-reach max score $(\mathrm{cm})$ & 9620 & $28.1 \pm 8.4$ & 4796 & $25.4 \pm 7.6$ & 4824 & $30.9 \pm 8.3$ & -0.69 & $-0.73,-0.65$ \\
\hline Total handgrip strength (kg) & 9668 & $33.5 \pm 9.4$ & 4815 & $34.5 \pm 9.6$ & 4853 & $32.6 \pm 9.2$ & 0.21 & $0.17,0.25$ \\
\hline PACER (20 m laps) & 9393 & $23.4 \pm 14.1$ & 4710 & $25.8 \pm 15.8$ & 4683 & $20.9 \pm 11.6$ & 0.35 & $0.31,0.40$ \\
\hline Plank time (sec) & 9606 & $61.8 \pm 43.8$ & 4780 & $62.4 \pm 44.8$ & 4826 & $61.3 \pm 42.9$ & 0.02 & $-0.02,0.06$ \\
\hline Body mass index $\left(\mathrm{kg} / \mathrm{m}^{2}\right)$ & 9455 & $19.0 \pm 3.8$ & 4716 & $18.9 \pm 3.9$ & 4739 & $19.0 \pm 3.7$ & -0.01 & $-0.05,0.03$ \\
\hline Waist circumference $(\mathrm{cm})$ & 9395 & $67.3 \pm 10.8$ & 4677 & $67.4 \pm 11.1$ & 4718 & $67.2 \pm 10.6$ & 0.02 & $-0.02,0.07$ \\
\hline CAMSA max score (/28) & 9488 & $20.6 \pm 3.9$ & 4752 & $21.0 \pm 3.9$ & 4736 & $20.3 \pm 3.8$ & -0.17 & $-0.21,-0.13$ \\
\hline Daily Behaviour score (/32) & 9783 & $18.6 \pm 7.7$ & 4867 & $18.6 \pm 7.9$ & 4916 & $18.5 \pm 7.4$ & -0.02 & $-0.06,0.02$ \\
\hline Daily steps taken & 6640 & $11,512 \pm 4006$ & 3088 & $12,355 \pm 4252$ & 3552 & $10,779 \pm 3624$ & 0.40 & $0.35,0.45$ \\
\hline Physical activity guideline adherence (days/week) & 9787 & $5.0 \pm 1.9$ & 4869 & $5.0 \pm 2.0$ & 4918 & $4.9 \pm 1.9$ & -0.07 & $-0.11,-0.04$ \\
\hline Daily screen time (hrs) & 9770 & $2.5 \pm 1.9$ & 4853 & $2.7 \pm 2.1$ & 4917 & $2.2 \pm 1.8$ & -0.30 & $-0.34,-0.26$ \\
\hline Daily non-screen sedentary time (hrs) & 9776 & $1.7 \pm 1.3$ & 4863 & $1.6 \pm 1.3$ & 4913 & $1.7 \pm 1.3$ & 0.08 & $0.04,0.12$ \\
\hline Motivation and Confidence score (/18) & 9625 & $12.5 \pm 2.7$ & 4778 & $12.7 \pm 2.8$ & 4847 & $12.2 \pm 2.6$ & 0.18 & $0.14,0.22$ \\
\hline Adequacy (/28) & 9628 & $21.9 \pm 4.3$ & 4779 & $22.3 \pm 4.3$ & 4849 & $21.4 \pm 4.2$ & -0.22 & $-0.26,-0.18$ \\
\hline Predilection (/36) & 9628 & $28.8 \pm 5.9$ & 4779 & $29.0 \pm 6.0$ & 4849 & $28.7 \pm 5.8$ & -0.05 & $-0.09,-0.01$ \\
\hline Benefits-to-barriers ratio (/4) & 9746 & $1.6 \pm 1.2$ & 4855 & $1.6 \pm 1.2$ & 4891 & $1.5 \pm 1.1$ & -0.12 & $-0.16,-0.08$ \\
\hline Physical activity level compared to peers $(/ 10)$ & 9865 & $7.2 \pm 2.1$ & 4906 & $7.3 \pm 2.2$ & 4959 & $7.0 \pm 2.0$ & 0.12 & $0.08,0.16$ \\
\hline Skill level compared to peers $(/ 10)$ & 9863 & $6.7 \pm 2.3$ & 4906 & $7 \pm 2.4$ & 4957 & $6.4 \pm 2.2$ & 0.27 & $0.23,0.31$ \\
\hline Knowledge and Understanding score (/18) & 9797 & $12.0 \pm 2.7$ & 4870 & $11.8 \pm 2.8$ & 4927 & $12.2 \pm 2.6$ & 0.11 & $0.07,0.15$ \\
\hline Minutes of daily MVPA (/1) & 9833 & $0.6 \pm 0.5$ & 4892 & $0.7 \pm 0.5$ & 4941 & $0.6 \pm 0.5$ & 0.15 & $0.11,0.19$ \\
\hline Minutes of daily screen time (/1) & 9837 & $0.2 \pm 0.4$ & 4893 & $0.2 \pm 0.4$ & 4944 & $0.1 \pm 0.3$ & -0.17 & $-0.21,-0.13$ \\
\hline Cardiorespiratory fitness definition (/1) & 9822 & $0.6 \pm 0.5$ & 4882 & $0.5 \pm 0.5$ & 4940 & $0.6 \pm 0.5$ & 0.11 & $0.07,0.15$ \\
\hline Muscular endurance definition (/1) & 9829 & $0.7 \pm 0.4$ & 4890 & $0.7 \pm 0.4$ & 4939 & $0.8 \pm 0.4$ & 0.10 & $0.06,0.13$ \\
\hline Healthy definition (/5) & 9856 & $4.0 \pm 0.9$ & 4908 & $3.9 \pm 1.0$ & 4948 & $4.0 \pm 0.9$ & -0.18 & $-0.22,-0.14$ \\
\hline Fill in the missing words (/5) & 9814 & $3.6 \pm 1.5$ & 4880 & $3.6 \pm 1.5$ & 4934 & $3.6 \pm 1.4$ & 0.02 & $-0.02,0.06$ \\
\hline Safety gear during physical activity (/1) & 9856 & $0.3 \pm 0.3$ & 4908 & $0.3 \pm 0.3$ & 4948 & $0.4 \pm 0.3$ & -0.35 & $-0.39,-0.31$ \\
\hline How to get better at a sport skill (/1) & 9798 & $0.5 \pm 0.5$ & 4871 & $0.5 \pm 0.5$ & 4927 & $0.5 \pm 0.5$ & 0.03 & $-0.01,0.07$ \\
\hline How to improve physical fitness (/1) & 9804 & $0.8 \pm 0.4$ & 4872 & $0.8 \pm 0.4$ & 4932 & $0.8 \pm 0.4$ & 0.08 & $0.04,0.12$ \\
\hline Preferred leisure-time activity (/1) & 9835 & $0.7 \pm 0.4$ & 4894 & $0.7 \pm 0.5$ & 4941 & $0.8 \pm 0.4$ & -0.14 & $-0.17,-0.10$ \\
\hline Physical literacy score (/100) & 9781 & $62.7 \pm 12.2$ & 4866 & $63.1 \pm 13.0$ & 4915 & $62.2 \pm 11.3$ & -0.07 & $-0.11,-0.03$ \\
\hline
\end{tabular}

CAMSA Canadian Agility and Movement Skill Assessment, CAPL Canadian Assessment of Physical Literacy, Cl confidence interval, MVPA moderate- to vigorous-intensity physical activity, PACER Progressive Aerobic Cardiovascular Endurance Run, RBC Royal Bank of Canada, SD standard deviation Important (>0.8) effect sizes are in bold

Absolute scores on Physical Competence measures were on average $28.1 \pm 8.4 \mathrm{~cm}$ for sit-and-reach flexibility, $33.5 \pm 9.4 \mathrm{~kg}$ for handgrip strength (right + left), $23.4 \pm 14.1$ laps for the PACER shuttle run, $61.8 \pm 43.8 \mathrm{~s}$ for the isometric plank, $19.0 \pm 3.8 \mathrm{~kg} / \mathrm{m}^{2}$ for BMI, $67.3 \pm$ $10.8 \mathrm{~cm}$ for waist circumference, and $20.6 \pm 3.9$ out of 28 points for the CAMSA. Scores for handgrip strength, PACER, plank, and CAMSA were higher in boys than girls, and in older children than in younger children. Girls and younger children had better scores on sit-and-reach flexibility compared to boys and older children, respectively. The largest differences between boys and girls in the Physical Competence domain were for sit and reach (25.4 \pm 7.6 vs. $30.9 \pm 8.3 \mathrm{~cm}$, respectively; Cohen's $d=0.69$ ), the PACER $(25.8 \pm 15.8$ vs. $20.9 \pm 11.6$ laps, respectively; Cohen's $d=0.35$ ), and handgrip strength (34.5 \pm 9.6 vs. $32.6 \pm 9.2 \mathrm{~kg}$, respectively; Cohen's $d=0.21$ ). The rest of the effect sizes between genders for the Physical Competence domain measures were considered negligible (Cohen's $d$ range: 0.01 to 0.17 ). 


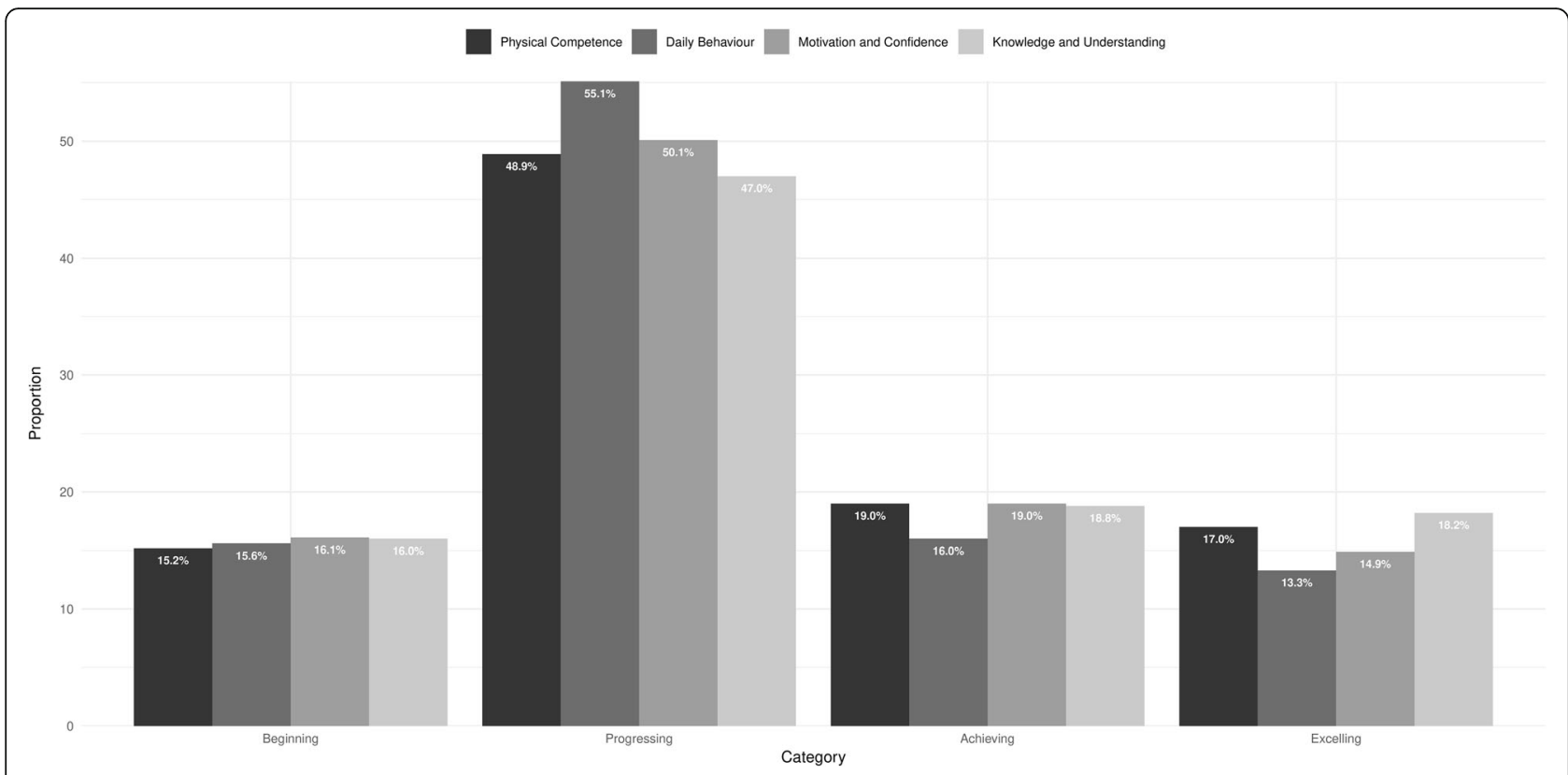

Fig. 2 Proportion of participants in each of the four interpretation categories by domain

For the Daily Behaviour domain, daily step counts were higher in boys than in girls $(12,355 \pm 4252$ vs. $10,779 \pm 3624$ steps; Cohen's $d=0.40$ ) and decreased with age. Self-reported physical activity (i.e., number of days adhering to $60 \mathrm{~min}$ of moderate- to vigorous-intensity physical activity [MVPA] per day) was similar across genders and ages (approximately 5 out of 7 days per week). Boys reported more screen time than girls $(2.7 \pm 2.1$ vs. $2.2 \pm 1.8 \mathrm{~h}$; Cohen's $d=0.30)$, with screen time increasing with age.

For the measures in the Motivation and Confidence domain, the effect sizes between genders were considered negligible to small (Cohen's $d$ range: 0.05 to 0.22 ) and no age-related differences were observed. With regard to the measures in the Knowledge and Understanding domain, all of the effect sizes between boys and girls were considered negligible (Cohen's $d$ range: 0.02 to 0.18), except for the question pertaining to safety gear while being physically active, where there was a small effect (Cohen's $d=0.35$ ), with girls outperforming boys. Overall, there was a pattern showing that the measures within the Knowledge and Understanding domain increased with age (effect size estimates range from 0.03 to 0.21 ) (see Additional file 2).

Additional file 2 provides the overall descriptive statistics stratified by age and gender. The percentiles for the total physical literacy score, domain scores, the individual components in the Physical Competence domain, and daily step counts are provided in Additional file 3.

Paradata from the RBC Learn to Play-CAPL project found that in a subset of 510 participants from six sites with detailed information about the protocol completion,
$5 \%$ or less of the participants refused to complete one or more CAPL protocols. Among the CAPL protocols, rates of refusal for the PACER, waist circumference, pedometer step, and weight protocols were similar, ranging from 3.7 to $5.4 \%$. Refusals occurred among 2 to $3 \%$ of participants for the plank, sit and reach, height, handgrip, and CAMSA protocols. Only three children $(0.05 \%)$ among this subset refused to complete the questionnaire protocols. A detailed analysis of missing data from the RBC Learn to Play-CAPL project is provided in the paper by Delisle Nyström et al. [33].

Although participants were not required to disclose personal information related to disabilities or medical conditions, parents or legal guardians were asked to indicate if a doctor had said that there were some types of physical activity that their child should not perform. Among a subset of 1196 ( $n=586,49 \%$ boys, mean age 10.1 years) participants with detailed participation data, 104 children $(9 \%)$ had disabilities or medical conditions identified by their parents. The disabilities/medical conditions reported included asthma/breathing conditions $(n=50,4 \%)$, developmental disability $(n=12,1 \%)$, physical or vision disability $(n=10,1 \%)$, learning disability/ attention deficit hyperactivity disorder $(n=8,1 \%)$, concussion/bleeding disorder $(\mathrm{n}=8,1 \%)$, heart condition $(n$ $=4,0.3 \%)$, arthritis $(n=2,0.2 \%)$, migraine $(n=2,0.2 \%)$, epilepsy/seizure risk $(n=2,0.2 \%)$, and other medical conditions $(n=6,0.5 \%)$.

All study sites were required to immediately report adverse events (e.g., injuries or illness) to the study coordinating centre. Of the 10,034 participants in the RBC Learn to Play-CAPL project, there were only two reported 
Table 3 RBC Learn to Play-CAPL descriptive statistics, by age

\begin{tabular}{|c|c|c|c|c|c|c|c|c|c|c|}
\hline \multirow[t]{2}{*}{ Variable } & \multicolumn{2}{|c|}{8 years } & \multicolumn{2}{|c|}{9 years } & \multicolumn{2}{|c|}{10 years } & \multicolumn{2}{|c|}{11 years } & \multicolumn{2}{|c|}{12 years } \\
\hline & $n$ & Mean \pm SD & $n$ & Mean \pm SD & $n$ & Mean \pm SD & $n$ & Mean $\pm S D$ & $n$ & Mean \pm SD \\
\hline Age (years) & 1117 & $8.5 \pm 0.3$ & 1958 & $9.5 \pm 0.3$ & 2488 & $10.5 \pm 0.3$ & 3222 & $11.5 \pm 0.3$ & 1144 & $12.3 \pm 0.2$ \\
\hline Physical Competence score (/32) & 1068 & $18.4 \pm 4.0$ & 1851 & $19.0 \pm 4.3$ & 2343 & $19.3 \pm 4.3$ & 3033 & $20.2 \pm 4.4$ & 1053 & $21.0 \pm 4.5$ \\
\hline Sit-and-reach max score $(\mathrm{cm})$ & 1088 & $29.1 \pm 7.3$ & 1878 & $28.7 \pm 8.0$ & 2379 & $28.0 \pm 8.2$ & 3096 & $27.8 \pm 8.7$ & 1082 & $28.0 \pm 9.2$ \\
\hline Total handgrip strength (kg) & 1087 & $26.2 \pm 6.0$ & 1875 & $29.5 \pm 7.0$ & 2414 & $32.3 \pm 8.0$ & 3108 & $36.6 \pm 9.1$ & 1088 & $41.4 \pm 10.6$ \\
\hline PACER (20 m laps) & 1045 & $20.7 \pm 13.0$ & 1833 & $21.8 \pm 12.9$ & 2341 & $22.4 \pm 13.3$ & 3032 & $24.7 \pm 14.6$ & 1047 & $27.1 \pm 16.1$ \\
\hline Plank time (sec) & 1080 & $54.7 \pm 39.5$ & 1869 & $61.3 \pm 45.5$ & 2391 & $60.5 \pm 42.7$ & 3093 & $64.3 \pm 45.0$ & 1077 & $65.1 \pm 42.8$ \\
\hline Body mass index $\left(\mathrm{kg} / \mathrm{m}^{2}\right)$ & 1064 & $17.3 \pm 2.8$ & 1860 & $18.3 \pm 3.5$ & 2378 & $18.9 \pm 3.7$ & 3051 & $19.6 \pm 3.9$ & 1058 & $20.0 \pm 4.1$ \\
\hline Waist circumference (cm) & 1075 & $61.3 \pm 7.9$ & 1837 & $64.4 \pm 9.6$ & 2349 & $67.2 \pm 10.4$ & 3036 & $69.8 \pm 11.1$ & 1054 & $71.3 \pm 11.0$ \\
\hline CAMSA max score (/28) & 1076 & $18 \pm 4.1$ & 1846 & $19.7 \pm 3.8$ & 2357 & $20.5 \pm 3.7$ & 3068 & $21.7 \pm 3.5$ & 1046 & $22.3 \pm 3.5$ \\
\hline Daily Behaviour score (/32) & 1089 & $19.6 \pm 7.4$ & 1911 & $19.7 \pm 7.4$ & 2461 & $18.6 \pm 7.5$ & 3206 & $17.7 \pm 7.7$ & 1112 & $17.7 \pm 8.2$ \\
\hline Daily steps taken & 706 & $12,160 \pm 3923$ & 1268 & $12,094 \pm 4019$ & 1727 & $11,578 \pm 4069$ & 2163 & $11,078 \pm 3919$ & 728 & $10,920 \pm 3972$ \\
\hline $\begin{array}{l}\text { Physical activity guideline } \\
\text { adherence (days/week) }\end{array}$ & 1092 & $4.8 \pm 2.2$ & 1913 & $5.0 \pm 2.0$ & 2461 & $5.0 \pm 1.9$ & 3205 & $4.9 \pm 1.9$ & 1112 & $4.9 \pm 1.8$ \\
\hline Daily screen time (hrs) & 1086 & $2.2 \pm 2.0$ & 1905 & $2.3 \pm 2.0$ & 2459 & $2.4 \pm 1.9$ & 3204 & $2.6 \pm 1.9$ & 1112 & $2.7 \pm 2.0$ \\
\hline $\begin{array}{l}\text { Daily non-screen sedentary } \\
\text { time (hrs) }\end{array}$ & 1080 & $1.4 \pm 1.3$ & 1914 & $1.5 \pm 1.3$ & 2458 & $1.6 \pm 1.3$ & 3206 & $1.8 \pm 1.3$ & 1114 & $1.9 \pm 1.3$ \\
\hline $\begin{array}{l}\text { Motivation and Confidence } \\
\text { score (/18) }\end{array}$ & 1058 & $12.3 \pm 2.5$ & 1873 & $12.6 \pm 2.5$ & 2405 & $12.6 \pm 2.7$ & 3161 & $12.4 \pm 2.8$ & 1124 & $12.5 \pm 3.0$ \\
\hline Adequacy (/28) & 1061 & $21.7 \pm 4.0$ & 1877 & $21.9 \pm 4.1$ & 2403 & $22.0 \pm 4.3$ & 3159 & $21.7 \pm 4.4$ & 1123 & $22.1 \pm 4.5$ \\
\hline Predilection (/36) & 1061 & $28.5 \pm 5.7$ & 1877 & $29.0 \pm 5.7$ & 2403 & $29.1 \pm 5.9$ & 3159 & $28.6 \pm 6.0$ & 1123 & $28.7 \pm 6.2$ \\
\hline Benefits-to-barriers ratio (/4) & 1073 & $1.5 \pm 1.3$ & 1900 & $1.6 \pm 1.2$ & 2450 & $1.6 \pm 1.2$ & 3193 & $1.6 \pm 1.1$ & 1127 & $1.6 \pm 1.2$ \\
\hline $\begin{array}{l}\text { Physical activity level compared } \\
\text { to peers }(/ 10)\end{array}$ & 1102 & $7.5 \pm 2.2$ & 1926 & $7.3 \pm 2.1$ & 2474 & $7.2 \pm 2.1$ & 3217 & $7.0 \pm 2.0$ & 1142 & $7.1 \pm 2.1$ \\
\hline Skill level compared to peers (/10) & 1102 & $7.0 \pm 2.5$ & 1925 & $6.9 \pm 2.4$ & 2475 & $6.7 \pm 2.3$ & 3216 & $6.5 \pm 2.2$ & 1141 & $6.7 \pm 2.2$ \\
\hline $\begin{array}{l}\text { Knowledge and Understanding } \\
\text { score (/18) }\end{array}$ & 1092 & $10.4 \pm 2.7$ & 1916 & $11.2 \pm 2.6$ & 2461 & $12.1 \pm 2.7$ & 3210 & $12.7 \pm 2.6$ & 1115 & $12.9 \pm 2.5$ \\
\hline Minutes of daily MVPA (/1) & 1099 & $0.5 \pm 0.5$ & 1927 & $0.5 \pm 0.5$ & 2471 & $0.6 \pm 0.5$ & 3216 & $0.7 \pm 0.5$ & 1117 & $0.7 \pm 0.4$ \\
\hline Minutes of daily screen time (/1) & 1103 & $0.1 \pm 0.3$ & 1927 & $0.1 \pm 0.3$ & 2468 & $0.2 \pm 0.4$ & 3217 & $0.2 \pm 0.4$ & 1118 & $0.2 \pm 0.4$ \\
\hline $\begin{array}{l}\text { Cardiorespiratory fitness } \\
\text { definition (/1) }\end{array}$ & 1101 & $0.4 \pm 0.5$ & 1919 & $0.5 \pm 0.5$ & 2466 & $0.6 \pm 0.5$ & 3214 & $0.7 \pm 0.5$ & 1118 & $0.7 \pm 0.5$ \\
\hline Muscular endurance definition (/1) & 1102 & $0.6 \pm 0.5$ & 1925 & $0.7 \pm 0.5$ & 2467 & $0.8 \pm 0.4$ & 3213 & $0.8 \pm 0.4$ & 1118 & $0.8 \pm 0.4$ \\
\hline Healthy definition (/5) & 1107 & $3.9 \pm 1.0$ & 1933 & $3.9 \pm 0.9$ & 2476 & $4.0 \pm 0.9$ & 3217 & $4.0 \pm 1.0$ & 1118 & $4.0 \pm 0.9$ \\
\hline Fill in the missing words (/5) & 1098 & $2.8 \pm 1.5$ & 1921 & $3.2 \pm 1.4$ & 2468 & $3.6 \pm 1.4$ & 3208 & $3.9 \pm 1.4$ & 1116 & $4.0 \pm 1.3$ \\
\hline $\begin{array}{l}\text { Safety gear during physical } \\
\text { activity }(/ 1)\end{array}$ & 1107 & $0.3 \pm 0.3$ & 1933 & $0.3 \pm 0.3$ & 2476 & $0.3 \pm 0.3$ & 3217 & $0.3 \pm 0.3$ & 1118 & $0.3 \pm 0.3$ \\
\hline How to get better at a sport skill (/1) & 1092 & $0.4 \pm 0.5$ & 1918 & $0.5 \pm 0.5$ & 2460 & $0.5 \pm 0.5$ & 3210 & $0.5 \pm 0.5$ & 1114 & $0.6 \pm 0.5$ \\
\hline How to improve physical fitness (/1) & 1092 & $0.7 \pm 0.5$ & 1921 & $0.8 \pm 0.4$ & 2462 & $0.8 \pm 0.4$ & 3211 & $0.8 \pm 0.4$ & 1114 & $0.8 \pm 0.4$ \\
\hline Preferred leisure-time activity (/1) & 1098 & $0.6 \pm 0.5$ & 1928 & $0.7 \pm 0.5$ & 2473 & $0.7 \pm 0.4$ & 3214 & $0.7 \pm 0.4$ & 1118 & $0.7 \pm 0.4$ \\
\hline Physical literacy score (/100) & 1093 & $60.9 \pm 11.2$ & 1915 & $62.4 \pm 11.5$ & 2456 & $62.6 \pm 12.2$ & 3202 & $62.9 \pm 12.5$ & 1112 & $64.2 \pm 13.5$ \\
\hline
\end{tabular}

CAMSA Canadian Agility and Movement Skill Assessment, CAPL Canadian Assessment of Physical Literacy, MVPA moderate- to vigorous-intensity physical activity, PACER Progressive Aerobic Cardiovascular Endurance Run, RBC Royal Bank of Canada, SD standard deviation

adverse events. One child twisted their ankle when reversing direction in the PACER shuttle run, and another child had a similar injury when they stepped on a hoop during the CAMSA assessment. Both incidents were minor injuries.

\section{Discussion}

This is the first study to report the physical literacy levels of a large sample of Canadian children. Overall, there were no large differences between boys' and girls' total physical literacy scores or the individual domain 
scores. Using the CAPL's interpretation system [34], the results show that on average the total physical literacy score, as well as the Physical Competence, Daily Behaviour, and Motivation and Confidence domains are at the "progressing" level, and only the Knowledge and Understanding domain is at the desired "achieving" level. These overall "low" scores could be due to societal change where, from a young age, children's free time is more focused on screens than active play. The reduced active play time could influence the scores for overall physical literacy as well as for the Physical Competence, Daily Behaviour, and Motivation and Confidence domains, as children are not developing the skills needed to adequately achieve in these areas. It is important to note that the interpretation of the total score and the domain scores is based upon cut-points informed by criterion thresholds for measures where such thresholds exist (e.g., step counts, BMI), and upon normative thresholds believed to be consistent with current trends in obesity, fitness, and physical inactivity when criterion thresholds were not available. Future research needs to further validate these thresholds. However, the average values and the overall classification of "progressing" for the total physical literacy score and three of the four domain scores demonstrates that there is room for improvement in Canadian children's physical literacy, and that greater efforts for the promotion of physical literacy are needed.

\section{Physical competence}

For the individual measures within the Physical Competence domain, we found boys had higher scores than girls for handgrip strength, PACER, plank, and CAMSA; whereas girls scored higher on the sit-and-reach measure. For handgrip strength, a previous Canadian study also found that boys scored higher on handgrip strength than girls [16]. However, the children in the RBC Learn to Play-CAPL project had higher mean values than the children from the Canadian Health Measures Survey (CHMS) 2007-2009 [16] (boys: $34.5 \mathrm{~kg}$ vs. $25 \mathrm{~kg}$, respectively; and girls: $32.6 \mathrm{~kg}$ vs. $23 \mathrm{~kg}$, respectively). The difference in the mean values is probably due to the age differences in the two studies (8- to 12-year-olds in the RBC Learn to Play-CAPL study and 6- to 10-year-olds in the CHMS). Using the sex- and age-specific cut-points created by Tomkinson et al. [35] for the number of completed laps in the 20-m shuttle run, boys aged 9-12 years from the RBC Learn to Play-CAPL study would be categorized in the 30th percentile; whereas girls would be classified between the 20th and $<40$ th percentiles. Using the quintile framework, this would classify both boys and girls of all age categories as having low cardiorespiratory endurance [35]. For sit-and-reach flexibility, the CHMS 2007-2009 [16] also found that girls had higher scores than boys, with similar results being observed between both studies. To date, there are no comparative data for the plank or CAMSA; therefore, the RBC Learn to Play-CAPL project is providing baseline measures for these important indicators of physical competence from a large sample of Canadian children.

Using the RBC Learn to Play-CAPL data, Lang et al. [36] found positive relationships between physical literacy and cardiorespiratory fitness. Furthermore, a positive reciprocal relationship between physical activity and motor competence has been demonstrated across children and youth aged 6 to 13 years, with further evidence of a mediating effect of aerobic fitness $\left(\mathrm{VO}_{2}\right.$ peak) in both directions [37]. These findings support the development and promotion of interventions that target each of these domains of physical literacy to activate positive feedback loops amongst the domains and to facilitate physical literacy development.

The two anthropometric measurements included in the Physical Competence domain were BMI and waist circumference, with negligible differences being observed between genders for both indicators. Using the WHO's BMI-for-age percentiles [25], which are specific for sex and age, we found that boys and girls of all ages were within or close to being within the 75th and 85th percentile, which would classify the children on the higher end of normal weight on average. With regard to waist circumference, using the Centers for Disease Control and Prevention age- and sex-specific reference values [38], boys and girls would be classified on average as normal. Boys aged 8-12 years were between the 50th and 75th percentile, with 11-year-olds being just below the 50th percentile. For girls, 8-year-olds were between the 50th and 75th percentile whereas girls aged 912 years were between the 25th and 50th percentiles.

\section{Daily behaviour}

The individual measures within the Daily Behaviour domain showed a small difference between boys and girls for average daily step counts and daily screen time, with boys accumulating more steps and more screen time than girls. Furthermore, it was observed that daily step counts decreased with age in both boys and girls. Our findings agree with those from the CHMS (compiled data from 2007 to 2009, 2009-2011, 2012-2013, and 2014-2015 cycles), where they also found similar patterns using objectively measured physical activity data [39]. The average step counts for boys and girls in the RBC Learn to Play-CAPL project was 12,355 and 10,779 steps, respectively, with only $27 \%$ of boys and $14 \%$ of girls meeting the 12,000 recommended steps per day, which is equivalent to $60 \mathrm{~min}$ of MVPA $[40,41]$. The proportion meeting the 12,000 step recommendation is slightly lower than that from the Physical Activity Levels 
Among Youth study (41\% of 5- to 19-year-olds; 20142016 data) from the Canadian Fitness and Lifestyle Research Institute [41]. Further, the average values obtained in this study are slightly lower than those obtained in the 2007-2009 CHMS, where boys and girls had on average 13,217 and 11,745 steps, respectively [42]. As stated previously, the observed differences are possibly due to the age differences between the two studies: 8- to 12-year-olds in RBC Learn to Play-CAPL and 6- to 10-year-olds in CHMS.

Boys and girls participating in the RBC Learn to PlayCAPL project reported being engaged in screen-based activities for on average 2.7 and $2.2 \mathrm{~h} /$ day, respectively, which exceeds the recommendation of $\leq 2 \mathrm{~h}$ of recreational screen time daily [43]. In the 2009-2011 and 2012-2013 CHMS [44], boys and girls aged 5 to 11 years reported (with parental assistance) a similar amount of screen time (2.4 and $2.3 \mathrm{~h} /$ day, respectively) compared to the participants in the present study. Using the information from RBC Learn to Play-CAPL participants who had provided complete and valid pedometer data, Belanger et al. [45] found that $20 \%$ of children met the physical activity guidelines and 57\% met screen time recommendations. Given the consistently low daily levels of physical activity and excess screen time found here and reported in other studies, it is clear that more health promotion and policy work needs to be done to improve the Daily Behaviour domain of children's physical literacy.

\section{Knowledge and understanding}

The physical literacy knowledge questionnaire was based on existing physical and health education curricula, and specifically developed for use within the RBC Learn to Play-CAPL project; therefore, no comparative data exist. Longmuir et al. [26] investigated the feasibility, validity, and reliability of the questionnaire within a subset of the RBC Learn to Play-CAPL participants and concluded that it was a feasible, valid, and reliable tool to assess knowledge in 8- to 12-year-old Canadian children. Overall, the Knowledge and Understanding domain score did not differ by gender; however, an increase in the domain score was observed with age, as might be expected. $\mathrm{Nu}$ merous governments and public health agencies have been working on increasing the public's knowledge regarding the amount of physical activity that is needed for health benefits, with the hope that greater knowledge will lead to better decisions regarding physical activity. Very few studies have been conducted to date investigating children's knowledge of physical activity guidelines and their physical activity levels. A study by Best et al. [46] found that knowledge of the physical activity guidelines was not an important predictor of physical activity in children and youth aged 11 to 16 years. However, another study by $\mathrm{Xu}$ et al. [47] found that Chinese children in grades 4 through 7 who became more aware of the relationships between obesity and physical activity significantly increased the frequency and amount of time spent on physical activity. Given the mixed evidence regarding the associations between knowledge of physical activity guidelines and actual physical activity levels, this area warrants further investigation.

\section{Motivation and confidence}

Motivation is an important predictor and self-efficacy an important correlate of physical activity in children and youth $[48,49]$. Within the Motivation and Confidence domain, negligible to small effect sizes were observed in the RBC Learn to Play-CAPL dataset for the individual measures between genders, and no age-related differences were observed. Participants' perceived levels of adequacy and predilection for physical activity were moderately related to cardiorespiratory fitness [50], which has also been observed in another study [51]. These findings lend support to the importance of considering psychological factors when creating physical literacy interventions.

\section{Strengths and limitations}

The CAPL is a large field-based assessment battery that includes 25 measures within four domains. A total of 10,034 children participated in the RBC Learn to PlayCAPL project; however, due to the large number of assessments, there were a lot of missing data. Delisle Nyström et al. [33] conducted an exploratory analysis of these missing data, and found that the pedometer step counts accounted for the greatest source of missing data (33.8\%), followed by the components of the Physical Competence domain (3.6-6.4\%), and the CSAPPA subscales (4.0\%). To reduce the burden of the CAPL battery of tests, Gunnell et al. [52] conducted factor analyses to create a shorter and more theoretically aligned CAPL version. Through this work it was found that CAPL could be reduced to 14 indicators across the same four domains, and the revised version is now referred to as CAPL-2 [53].

This is the first study to provide descriptive and normative percentile data for physical literacy from a large sample of Canadian children, providing a baseline to be used for future comparisons, informing policy, and assessing interventions. The findings are strengthened by the large and diverse sample size, the reliable and valid protocols that were used to assess physical literacy $[20,24,26]$, the standardized methods used in data collection, and the ability of the CAPL to assess physical literacy without bias across children aged 8 to 12 years [54] and in varying weight classes [55]. A limitation of the study was the use of convenience sampling and therefore the findings may not be generalizable to all 
Canadian children aged 8 to 12 years. However, all sites were instructed to collect data in locations that offered varied levels of urbanization (i.e., rural, suburban, and urban) and socioeconomic status. Although not assessed, diversity is likely since most data were collected within schools, which reach a broad spectrum of children from across social classes and ethnicities. Nevertheless, information on socioeconomic status and cultural background was not collected, and this information may be important to interpretation of the findings. Future research should consider whether a maturation measure in the CAPL may provide additional insights on differences observed between genders and ages, especially when focusing on older children or adolescents. Despite these limitations, the characteristics of the RBC Learn to Play-CAPL sample were similar to nationally representative CHMS data (e.g., prevalence of overweight and obesity $36.4 \%$ [55] and 31.4\% [56], respectively).

\section{Conclusions}

These results provide the largest and most comprehensive assessment of the physical literacy of Canadian children to date, providing a "state of the nation" baseline. They can be used to monitor and inform domestic changes in this area of study going forward. Importantly, they highlight the need to enhance efforts to promote the physical literacy of Canadian children.

\section{Additional files}

Additional file 1: CAPL interpretation categories for each domain and each measure by age and gender. (DOCX $112 \mathrm{~kb}$ )

Additional file 2: RBC Learn to Play-CAPL descriptive statistics stratified by gender and age. (DOCX $50 \mathrm{~kb}$ )

Additional file 3: Percentiles for the total physical literacy score, domain scores, the individual components in the physical competence domain, and daily step counts. (DOCX $132 \mathrm{~kb}$ )

\section{Abbreviations \\ BMl: Body mass index; CAMSA: Canadian Agility and Movement Skill Assessment;" CAPL: Canadian Assessment of Physical Literacy; CHEO Rl: Children's Hospital of Eastern Ontario Research Institute; CHMS: Canadian Health Measures Survey; CSAPPA: Children's Self-Perceptions of Adequacy in, and Predilection for Physical Activity; GAMLSS: Generalized additive models for location, scale, and shape; ICC: Intraclass correlation coefficient; MVPA: Moderate- to vigorous- intensity physical activity; PACER: Progressive Aerobic Cardiovascular Endurance Run; Pl: Principal investigator; RBC: Royal Bank of Canada; WHO: World Health Organization}

\footnotetext{
Acknowledgements

The authors acknowledge the research funding support provided by the RBC Learn to Play-CAPL project and the Public Health Agency of Canada, delivered in partnership with ParticipACTION; the Mitacs Accelerate Program; and New Investigator and Canada Graduate Scholarship (Master's) awards from the Canadian Institute of Health Research. The authors are grateful to all Project Managers, Research Assistants, and Research Students who worked on the project at the sites across Canada. Finally, the authors express their gratitude to all of the study participants, school boards, and camp programs that supported the data collection.
}

\section{Funding}

This study was funded by RBC, the Public Health Agency of Canada, and Mitacs, and was delivered in partnership with ParticipACTION. Publication charges for this article have been funded by the RBC Learn to Play project and the Public Health Agency of Canada, delivered in partnership with ParticipACTION.

\section{Availability of data and materials}

The dataset(s) supporting the results of this article and additional files are available upon reasonable request to Mark S. Tremblay (mtremblay@cheo.on.ca) through a data user agreement with the CHEO RI.

\section{About this supplement}

This article has been published as part of BMC Public Health Volume 18 Supplement 2, 2018: Canadian Assessment of Physical Literacy. The full contents of the supplement are available online at https://bmcpublichealth. biomedcentral.com/articles/supplements/volume-18-supplement-2.

\section{Authors' contributions}

MST and PEL were responsible for the RBC Learn to Play-CAPL study design. MST was responsible for the conceptualization of this manuscript. PEL, KDA, $B B, J L C, M J G, N H, A M K, K N L, B L, D J M, L J M, T J S, D S, M R S$, and SJW led the data collection at the various sites. JDB prepared the data tables and figures. MST, CDN, and KB wrote the manuscript. All authors edited, reviewed, and approved the final manuscript.

\section{Ethics approval and consent to participate}

Ethics approval for this project was obtained from: Antigonish, NS - St. Francis University Research Ethics Board and the Strait Regional School Board; Calgary, AB - Mount Royal University Human Research Ethics Board; Charlottetown, PEl University of Prince Edward Island Research Ethics Board and the Prince Edward Island Public Schools Branch Research Ethics Board; Halifax, NS - Dalhousie University Research and Ethics Board, and the Halifax Regional School Board; Lethbridge, AB - University of Lethbridge Human Subject Research Committee; North Bay, ON - Nipissing University Research Ethics Board, Near North District School Board, Nipissing Parry Sound Catholic District School Board, and Conseil Scolaire Catholique Franco-Nord; Ottawa, ON - CHEO Research Ethics Board, University of Ottawa Research Ethics Board, Ottawa-Carleton District School Board, Ottawa Catholic School Board, Conseil des écoles catholiques du CentreEst, Conseil des écoles publiques de l'Est de l'Ontario, Upper Canada District School Board, Durham District School Board, University of Illinois at UrbanaChampaign; Trois-Rivières, QC - Université du Québec à Trois-Rivières Research Ethics Board; Victoria, BC - Camosun College Research Ethics Board and the Greater Victoria School District; Windsor, ON - University of Windsor Research Ethics Board and the Windsor Essex Catholic District School Board; Winnipeg, MB - The University of Winnipeg University Human Research Ethics Board, River East Transcona School Division, and St. James-Assiniboia School Division. Written informed consent was obtained from parents or legal guardians, and participating children also provided verbal assent.

\section{Consent for publication}

Not applicable.

\section{Competing interests}

The authors declare that they have no competing interests.

\section{Publisher's Note}

Springer Nature remains neutral with regard to jurisdictional claims in published maps and institutional affiliations.

\footnotetext{
Author details

'Healthy Active Living and Obesity Research Group, Children's Hospital of Eastern Ontario Research Institute, 401 Smyth Road, Ottawa, ON K1H 8L1, Canada. ${ }^{2}$ Centre for Sport and Exercise Education, Camosun College, Victoria, BC V9E 2C1, Canada. ${ }^{3}$ School of Physical and Health Education, Nipissing University, North Bay, ON P1B 8L7, Canada. ${ }^{4}$ Department of Kinesiology and Physical Education, University of Lethbridge, Lethbridge, AB T1K 3M4, Canada. ${ }^{5}$ Department of Kinesiology and Applied Health, University of Winnipeg, Winnipeg, MB R3B 2E9, Canada. ${ }^{6}$ Department of Human Kinetics, St. Francis Xavier University, Antigonish, NS B2G 2W5, Canada. ${ }^{7}$ Department of Applied Human Sciences, University of Prince Edward Island,
} 
Charlottetown, PEI C1A 4P3, Canada. ${ }^{8}$ School of Kinesiology and Health Studies, Queen's University, Kingston, ON K7L 3N6, Canada. ${ }^{9}$ Department of Health and Physical Education, Mount Royal University, Calgary, AB T3E 6K6, Canada. ${ }^{10} \mathrm{~S}$ chool of Health and Human Performance, Dalhousie University, Halifax, NS B3H 4R2, Canada. ${ }^{11}$ Department of Kinesiology, University of Windsor, Windsor, ON N9B 3P4, Canada.

\section{Published: 2 October 2018}

\section{References}

1. Tremblay MS, Costas-Bradstreet C, Barnes JD, Bartlett B, Dampier D, Lalonde C, et al. Canada's Physical Literacy Consensus Statement: process and outcome. BMC Public Health. 2018;18(Suppl 2) https://doi.org/10.1186/s12889-018-5903-X.

2. Tremblay MS, Lloyd M. Physical literacy measurement - the missing piece. PHE Journal. 2010;Spring:26-30

3. Delaney B, Donnelly P, News J. Improving physical literacy. Belfast: Sport Northern Ireland; 2008

4. Schools and Physical Activity Task and Finish Group. Physical literacy - an all-Wales approach to increasing levels of physical activity for children and young people. Cardiff: Government of Wales; 2013.

5. Poitras VJ, Gray CE, Borghese MM, Carson V, Chaput JP, Janssen I, et al. Systematic review of the relationships between objectively measured physical activity and health indicators in school-aged children and youth. Appl Physiol Nutr Metab. 2016;41 (6 Suppl 3):S197-239.

6. Castelli DM, Barcelona JM, Bryant L. Contextualizing physical literacy in the school environment: the challenges. J Sport Health Sci. 2015;4(2):156-63.

7. Edwards LC, Bryant AS, Keegan RJ, Morgan K, Jones AM. Definitions, foundations and associations of physical literacy: a systematic review. Sports Med. 2017;47(1):113-26.

8. Edwards LC, Bryant AS, Keegan RJ, Morgan K, Cooper SM, Jones AM. 'Measuring' physical literacy and related constructs: a systematic review of empirical findings. Sports Med. 2018;48(3):659-82.

9. Longmuir P. Understanding the physical literacy journey of children: the Canadian assessment of physical literacy. Bulletin of the International Council of Sport Science and Physical Education ICSSPE. 2013;65:1-6.

10. Longmuir PE, Boyer C, Lloyd M, Yang Y, Boiarskaia E, Zhu W, et al. The Canadian assessment of physical literacy: methods for children in grades 4 to 6 (8 to 12 years). BMC Public Health. 2015;15:767.

11. Francis $C E$, Longmuir $P E$, Boyer $C$, Andersen $L B$, Barnes JD, Boiarskaia E, et al. The Canadian assessment of physical literacy: development of a model of children's capacity for a healthy, active lifestyle through a Delphi process. J Phys Act Health. 2016;13(2):214-22.

12. Lloyd M, Colley RC, Tremblay MS. Advancing the debate on 'fitness testing' for children: perhaps we're riding the wrong animal. Pediatr Exerc Sci. 2010; 22(2):176-82.

13. Morrow JR, Fulton JE, Brener ND, Kohl HW. Prevalence and correlates of physical fitness testing in U.S. schools - 2000. Res Q Exerc Sport. 2008;79(2): $141-8$.

14. Rowland T. The horse is dead; let's dismount. Pediatr Exerc Sci. 1995;7:117-20.

15. Cale L, Harris J, Chen MH, Corbin C, Fox K, Morrow JR, Plowman SA. More than 10 years after "the horse is dead...": surely it must be time to "dismount"?! Pediatr Exerc Sci. 2007:19(2):115-31.

16. Tremblay MS, Shields M, Laviolette M, Craig CL, Janssen I, Connor GS. Fitness of Canadian children and youth: results from the 2007-2009 Canadian health measures survey. Health Rep. 2010;21(1):7-20.

17. Ortega FB, Ruiz JR, Castillo MJ, Sjostrom M. Physical fitness in childhood and adolescence: a powerful marker of health. Int J Obes. 2008:32(1):1-11.

18. ParticipACTION, Canadian Sport for Life Society, the Healthy Active Living and Obesity Research Group at the Children's Hospital of Eastern Ontario, Physical Health and Education Canada, Canadian Parks and Recreation Association, Ontario Society of Physical Activity Promoters in Public Health. Canada's Physical Literacy Consensus Statement. June 2015. https:// www.participaction.com/sites/default/files/downloads/ Consensus\%20Handout\%20-\%20ENG.pdf. Accessed 8 May 2018.

19. Brener ND, Kann L, Shanklin S, Kinchen S, Eaton DK, Hawkins J, et al. Methodology of the Youth Risk Behavior Surveillance System - 2013. MMWR Recomm Rep. 2013;62(RR-1):1-20.

20. Boyer C, Tremblay MS, Saunders TJ, McFarlane A, Borghese M, Lloyd M, et al. Feasibility, validity and reliability of the plank isometric hold as a field-based assessment of torso muscular endurance for children 8-12 years of age. Pediatr Exerc Sci. 2013;25(3):407-22.
21. Scott SN, Thompson DL, Coe DP. The ability of the PACER to elicit peak exercise response in youth [corrected]. Med Sci Sports Exerc. 2013;45(6): 1139-43.

22. Carrel AL, Bowser J, White D, Moberg DP, Weaver B, Hisgen J, et al. Standardized childhood fitness percentiles derived from school-based testing. J Pediatr. 2012;161(1):120-4.

23. Lander N, Morgan PJ, Salmon J, Logan SW, Barnett LM. The reliability and validity of an authentic motor skill assessment tool for early adolescent girls in an Australian school setting. J Sci Med Sport. 2017;20(6):590-4.

24. Longmuir PE, Boyer C, Lloyd M, Borghese MM, Knight E, Saunders TJ, et al. Canadian agility and movement skill assessment (CAMSA): validity, objectivity, and reliability evidence for children 8-12 years of age. J Sport Health Sci. 2017; 6(2):231-40.

25. World Health Organization. Growth reference 5-19 years. World Health Organization. 2007. www.who.int/growthref/who2007_bmi_for_age/en/. Accessed 8 May 2018.

26. Longmuir PE, Woodruff SJ, Boyer C, Lloyd M, Tremblay MS. Physical Literacy Knowledge Questionnaire: feasibility, validity, and reliability for Canadian children aged 8 to 12 years. BMC Public Health. 2018;18(Suppl 2) https://doi. org/10.1186/s1 2889-018-5890-y.

27. Tremblay MS, Warburton DE, Janssen I, Paterson DH, Latimer AE, Rhodes RE, et al. New Canadian physical activity guidelines. Appl Physiol Nutr Metab. 2011:36(1):36-46.

28. Tremblay MS, Leblanc AG, Janssen I, Kho ME, Hicks A, Murumets K, et al. Canadian sedentary behaviour guidelines for children and youth. Appl Physiol Nutr Metab. 2011;36(1):59-64.

29. Garcia AW, Broda MA, Frenn M, Coviak C, Pender NJ, Ronis DL. Gender and developmental differences in exercise beliefs among youth and prediction of their exercise behavior. J Sch Health. 1995;65(6):213-9.

30. Hay JA. Adequacy in and predilection for physical activity in children. Clin J Sport Med. 1992;2(3):192-201.

31. Rigby RA, Stasinopoulos DM. Generalized additive models for location, scale and shape, (with discussion). Appl Statist. 2005;54(3):507-54.

32. Cohen J. Statistical power analysis for the behavioral sciences. 2nd ed. New York: Lawrence Erlbaum Associates; 1988.

33. Delisle Nyström C, Barnes JD, Tremblay MS. An exploratory analysis of missing data from the Royal Bank of Canada (RBC) Learn to Play - Canadian Assessment of Physical Literacy (CAPL) project. BMC Public Health. 2018;18(Suppl 2) https:// doi.org/10.1186/s12889-018-5901-z.

34. Healthy Active Living and Obesity Research Group. Canadian assessment of physical literacy: manual for test administration. Healthy Active Living and Obesity Research Group; Children's Hospital of Eastern Ontario. 2013. https://www.capl-ecsfp.ca/capl-manual/. Accessed 8 May 2018.

35. Tomkinson GR, Lang JJ, Tremblay MS, Dale M, LeBlanc AG, Belanger K, et al. International normative $20 \mathrm{~m}$ shuttle run values from 1142026 children and youth representing 50 countries. Br J Sports Med. 2017;51(21):1545-54.

36. Lang JJ, Chaput J-P, Longmuir PE, Barnes JD, Belanger K, Tomkinson GR, et al. Cardiorespiratory fitness is associated with physical literacy in a large sample of Canadian children aged 8 to 12 years. BMC Public Health. 2018; 18(Suppl 2) https://doi.org/10.1186/s12889-018-5896-5.

37. Lima RA, Pfeiffer $K$, Larsen LR, Bugge A, Moller NC, Anderson LB, et al. Physical activity and motor competence present a positive reciprocal longitudinal relationship across childhood and early adolescence. J Phys Act Health. 2017; 14(6):440-7.

38. Fryar $C D, G u$ Q, Ogden $C L$, Flegal KM. Anthropometric reference data for children and adults: United States, 2011-2014. Vital Health Stat 3. 2016;3(39):1-46.

39. Colley RC, Carson V, Garriguet D, Janssen I, Roberts KC, Tremblay MS. Physical activity of Canadian children and youth, 2007 to 2015. Health Rep. 2017:28(10):8-16.

40. Colley RC, Janssen I, Tremblay MS. Daily step target to measure adherence to physical activity guidelines in children. Med Sci Sports Exerc. 2012;44(5):977-82.

41. ParticipACTION. Canadian kids need active bodies to build their best brains. The 2018 ParticipACTION report card on physical activity for children and youth. Toronto, ON: ParicipACTION. p. 2018.

42. Colley RC, Garriguet D, Janssen I, Craig CL, Clarke J, Tremblay MS. Physical activity of Canadian children and youth: accelerometer results from the 2007 to 2009 Canadian health measures survey. Health Rep. 2011:22(1):15-23.

43. Edelson LR, Mathias KC, Fulgoni VL, Karagounis LG. Screen-based sedentary behavior and associations with functional strength in 6-15 year-old children in the United States. BMC Public Health. 2016;16:116. 
44. Roberts KC, Yao X, Carson V, Chaput JP, Janssen I, Tremblay MS. Meeting the Canadian 24-hour movement guidelines for children and youth. Health Rep. 2017;28(10):3-7.

45. Belanger K, Barnes JD, Longmuir PE, Anderson KD, Bruner B, Copeland JL, et al. The relationship between physical literacy scores and adherence to Canadian physical activity and sedentary behaviour guidelines. BMC Public Health. 2018;18(Suppl 2) https://doi.org/10.1186/s12889-018-5897-4.

46. Best $P$, Tully MA, Corepal R, Kee F, Hunter RF. Time to 're-think' physical activity promotion for young people? Results from a repeated cross-sectional study. BMC Public Health. 2017;17(1):208.

47. Xu F, Wang X, Xiang D, Wang Z, Ye Q, Ware RS. Awareness of knowledge and practice regarding physical activity: a population-based prospective, observational study among students in Nanjing. China PLoS One. 2017; 12(6):e0179518.

48. Owen KB, Smith J, Lubans DR, Ng JY, Lonsdale C. Self-determined motivation and physical activity in children and adolescents: a systematic review and meta-analysis. Prev Med. 2014:67:270-9.

49. Bauman AE, Reis RS, Sallis JF, Wells JC, Loos RJ, Martin BW. Correlates of physical activity: why are some people physically active and others not? Lancet. 2012;380(9838):258-71.

50. MacDonald DJ, Saunders TJ, Longmuir PE, Barnes JD, Belanger K, Bruner B, et al. A cross-sectional study exploring the relationship between age, gender, and physical measures with adequacy in and predilection for physical activity. BMC Public Health. 2018;18(Suppl 2) https://doi.org/10. 1186/s12889-018-5893-8

51. Cairney J, Hay JA, Faught BE, Leger L, Mathers B. Generalized self-efficacy and performance on the 20-metre shuttle run in children. Am J Hum Biol. 2008;20(2):132-8.

52. Gunnell KE, Longmuir PE, Barnes JD, Belanger K, Tremblay MS. Refining the Canadian Assessment of Physical Literacy based on theory and factor analyses. BMC Public Health. 2018;18(Suppl 2) https://doi.org/10.1186/ s12889-018-5899-2.

53. Healthy Active Living and Obesity Research Group. Canadian assessment of physical literacy: manual for test administration second edition. Healthy Active Living and Obesity Research Group; Children's Hospital of Eastern Ontario. 2014. https://www.capl-eclp.ca/capl-manual/. Accessed 8 May 2018.

54. Dutil C, Tremblay MS, Longmuir PE, Barnes JD, Belanger K, Chaput J-P. Influence of the relative age effect on children's scores obtained from the Canadian Assessment of Physical Literacy. BMC Public Health. 2018; 18(Suppl 2) https://doi.org/10.1186/s12889-018-5895-6.

55. Delisle Nyström C, Traversy G, Barnes JD, Chaput J-P, Longmuir PE, Tremblay MS. Associations between domains of physical literacy by weight status in 8- to 12-year-old Canadian children. BMC Public Health. 2018;18(Suppl 2) https://doi.org/10.1186/s12889-018-5898-3.

56. Rao DP, Kropac E, Do MT, Roberts KC, Jayaraman GC. Childhood overweight and obesity trends in Canada. Health Promot Chronic Dis Prev Can. 2016; 36(9):194-8

Ready to submit your research? Choose BMC and benefit from:

- fast, convenient online submission

- thorough peer review by experienced researchers in your field

- rapid publication on acceptance

- support for research data, including large and complex data types

- gold Open Access which fosters wider collaboration and increased citations

- maximum visibility for your research: over $100 \mathrm{M}$ website views per year

At $\mathrm{BMC}$, research is always in progress.

Learn more biomedcentral.com/submissions 